\title{
Domain-wall coercivity in ferromagnetic systems with nonuniform local magnetic field
}

\author{
L. Půst \\ Institute of Physics, Academy of Sciences of the Czech Republic, Na Slovance 2, CZ - 18040 Praha 8, Czech Republic \\ G. Bertotti \\ Istituto Elettrotecnico Nazionale Galileo Ferraris, Corso Massimo d'Azeglio 42, I - 10125 Torino, Italy \\ I. Tomás \\ Institute of Physics, Academy of Sciences of the Czech Republic, Na Slovance 2, CZ - 18040 Praha 8, Czech Republic \\ G. Vértesy \\ Research Institute for Materials Science, Hungarian Academy of Sciences, H - 1525, P.O.B. 49, Budapest, Hungary
}

(Received 1 February 1996)

\begin{abstract}
Domain-wall (DW) coercive field, $H_{\mathrm{CW}}$, which characterizes pinning of DW's in soft magnetic materials, decreases strongly with increasing value of gradient, G, of the effective local DW-position-restoring magnetic field. Particular shapes of the dependence, $H_{\mathrm{CW}}(G)$, can be calculated from the mean energy dissipation of the DW moving over the particular profile of the DW pinning field, $H_{p}$. In this paper, $H_{\mathrm{CW}}(G)$ is calculated from a wall-pinning field, $H_{p}$, which is expressed as a stochastic function of the DW coordinate, $x_{\mathrm{DW}}$. The wall-pinning field, $H_{p}$, is described as a Wiener-Lévy stochastic process modified by two correlation lengths in such a way that $H_{p}$ is stationary for large DW displacements and $d H_{p} / d x_{\mathrm{DW}}$ is well defined for small DW displacements. The computed $H_{\mathrm{CW}}(G)$ is close to a hyperbolic decrease, but it approaches finite values if $G \rightarrow 0$ and it decreases in a much steeper way than $\propto 1 / G$ for high values of $G$, which agrees with the experimental observations. Experimentally, the dependence $H_{\mathrm{CW}}(G)$ was measured on close-packed arrays of cylindrical bubble domains in two thin films of magnetic garnets, where the local field gradient, $G$, was controlled within the range $10^{9}-10^{10} \mathrm{~A} / \mathrm{m}^{2}$ by changing distances between neighboring DW's. The DW coercive field, $H_{\mathrm{CW}}$, extrapolated from the measured values for $G \rightarrow 0$ was close to $80 \mathrm{~A} / \mathrm{m}$ for both samples, while $H_{\mathrm{CW}}\left(G \approx 10^{10} \mathrm{~A} / \mathrm{m}^{2}\right)$ was several times smaller. Fitting the calculated $H_{\mathrm{CW}}(G)$ dependence to the experimental data, we obtained values of the Wiener-Lévy correlation lengths well comparable to the DW width parameters. [S0163-1829(96)00941-1]
\end{abstract}

\section{INTRODUCTION}

Coercive properties of magnetic materials and the related width of their magnetic hysteresis loops are caused by several different magnetization mechanisms. The main ones are sudden flips over or rotation of the magnetization vector inside magnetically saturated volumes (domains and/or grains), generation and annihilation of magnetic domains and domain walls (DW's), and movement of the already existing domain walls through the nonuniform material. The latter process, namely the pinning and depinning of domain walls during their motion, is often dominant in soft magnetic materials.

The aim of the present work is to investigate the mechanism of the hysteretic behavior of the moving domain walls. In particular, we study the influence of nonuniform internal effective fields and/or of nonuniform external real fields applied at the wall position on the hysteretic properties of the wall pinning and depinning process. Both the experimental study and the accompanying theory are carried out for such a magnetic material, sample geometry and an applied field range that ensures the whole investigated change of the sample magnetic moment to be exclusively due to motion of $180^{\circ}$ DW's.

In our treatment, the internal DW position-restoring field, $H_{i}\left(x_{\mathrm{DW}}\right)$, experienced by the moving DW is described as

$$
H_{i}\left(x_{\mathrm{DW}}\right)=G x_{\mathrm{DW}}+H_{p}\left(x_{\mathrm{DW}}\right)
$$

where $x_{\mathrm{DW}}$ is the DW position and $H_{p}$ is the wall-pinning field expressed as a stochastic function of the DW position $x_{\mathrm{DW}}$. The term $G x_{\mathrm{DW}}$ is an odd function of $x_{\mathrm{DW}}$, and it is associated with the presence of some large-scale (compared to the mean period of the wall-pinning field $H_{p}$ ) parabolic energy well, which controls the average response of the DW's to external fields. We will often refer to it as the mean field gradient experienced by the DW. On the other hand, the wall-pinning field $H_{p}\left(x_{\mathrm{DW}}\right)$ is a random function of the DW position, describing the short-scale DW interaction with defects and other sources of the structural disorder. In Fig. 1 we see a schematic representation of the effective domain wall energy and its derivative (i.e., of the internal DW positionrestoring field $H_{i}$ vs the domain-wall position $x$ ), for low and high field gradients. In the frame of this description, the field gradient $G$ and the wall-pinning field $H_{p}\left(x_{\mathrm{DW}}\right)$ are the only physical quantities that determine the half-width, $H_{\mathrm{CW}}$, of the quasistatic hysteresis loop generated by the DW motion, which can be truly called the DW coercive field.

The calculation of $H_{\mathrm{CW}}$ is the basic problem in any description of the DW hysteresis and coercivity properties. ${ }^{1-3}$ In the literature, the large-scale wall-position-restoring forces acting on the domain structure [analogous to the term 


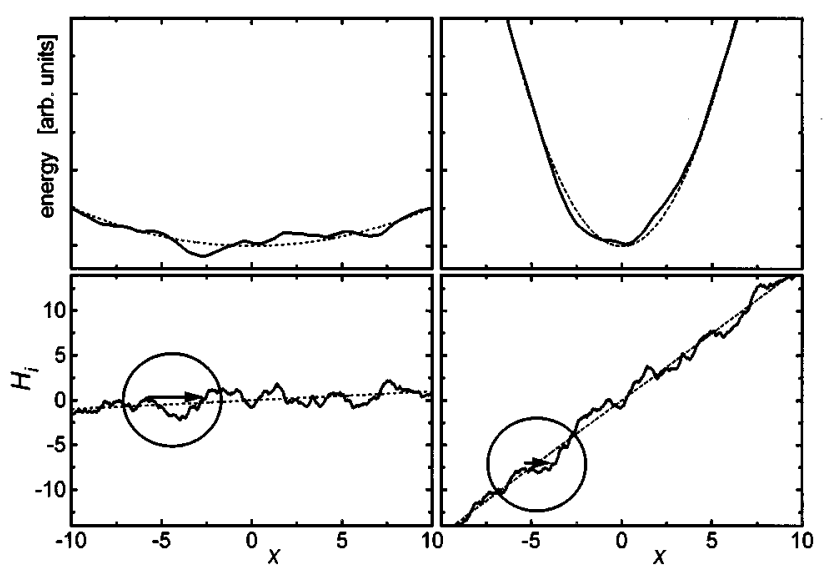

FIG. 1. Schematic representation of the domain structure energy (top) and of its derivative [i.e., of the related internal DW positionrestoring fields $H_{i}(x)$ ] (bottom) vs the dimensionless domain wall position, $x$, for low (left) and high (right) field gradient, $\beta$, respectively. The energy and the effective fields consist of two components: the short-range stochastic fluctuations due to the local wall pinning and the long-range parabolic energy well. The magnitude of the field gradient in the right figures is 15 times larger than in the left figures. It is obvious that the range of quasiequilibrium DW positions is much wider if domain wall is in low gradient (left) than if it is in high gradient (right). Therefore the hysteresis losses are lower for domain walls constrained at their positions by higher field gradients. Compare the corresponding Barkhausen jumps represented by the arrows in the bottom figures.

$G x_{\text {DW }}$ of Eq. (1)] are readily recognized to determine the permeability, $\mu$, of the system, but their possible influence on the value of the DW coercive field $H_{\mathrm{CW}}$ is rarely taken into consideration as it was done, e.g., in Refs. 4-7. Yet, this influence is by no means negligible and the principal aim of the present work is to extend the latest considerations and to give theoretical and experimental support to this statement. The basic point is that the DW coercive field $H_{\mathrm{CW}}$ is expected to be a decreasing function of the field gradient $G$.

The effect of the field gradient $G$ on the DW coercivity was measured directly by Grigorenko et al. ${ }^{7}$ on thin films of low magnetization magnetic garnets with large uniaxial anisotropy perpendicular to the film plane. A system of specially designed permanent magnets was applied for generation of the field gradient, $G$, around the position of a single DW, the magnitude of $G$ being changed by positioning of the pole pieces. An additional uniform field, $H_{a}$, was applied to move the DW. The DW coercive field $H_{\mathrm{CW}}$ was found to decrease approximately linearly with the field gradient $G$ up to $G \approx 2.2 \times 10^{9} \mathrm{~A} / \mathrm{m}^{2}$. The measured $H_{\mathrm{CW}}$ was equal to zero (within the experimental error) above this critical value, i.e., the observed DW moved without any measurable hysteresis loss.

Calculations based on a simple schematic description of the wall-pinning field $H_{p}$ led to the conclusion ${ }^{4,5,7}$ that the DW coercive field $H_{\mathrm{CW}}$ should be approximately a linear function of the field gradient $G$, of the form $H_{\mathrm{CW}}(G)$ $=H_{\mathrm{CW}}(0)-a G$, with $a$ being a suitable constant and $H_{\mathrm{CW}}(0)$ the coercive field of a DW free of any positionrestoring force.

Recently Půst and Bertotti ${ }^{6}$ calculated the dependence
$H_{\mathrm{CW}}(G)$ by a statistical approach, where the wall-pinning field $H_{p}\left(x_{\mathrm{DW}}\right)$ was described by a modified OrnsteinUhlenbeck process. They predicted the DW coercive field $H_{\mathrm{CW}} \propto 1 / G$, with suitable cutoffs at low and high values of the field gradient $G$. In the present work, this approach is developed in full detail, and the $H_{\mathrm{CW}}(G)$ dependence is determined by analytical and numerical methods, as a function of the statistical properties of the $H_{p}\left(x_{\mathrm{DW}}\right)$ stochastic process. The theoretical predictions are compared with the experimental $H_{\mathrm{CW}}(G)$ dependence measured on two magnetic garnet films with cylindrical bubble domain structure. In the experiments, the field gradient $G$ was changed in the range $10^{9}-10^{10} \mathrm{~A} / \mathrm{m}^{2}$ by modifying the bubble size and density. The comparison between the theory and the experiments leads to a quantitative estimate of the typical wavelength of the structural disorder space variations, which is in agreement with what is expected for the investigated materials.

The problem addressed in this article is of a general nature and it is at the heart of the hysteresis mechanisms. The actual origin of the effective restoring force, $G x_{\mathrm{DW}}$, is not important in determination of the coercivity reduction. It can be due to an externally applied real nonuniform field (as considered by Ref. 7), or to the general rate of change of the energy of the sample domain structure (as in the present experiments) or to the sample geometry, or to other causes. Only the magnitude of the field gradient $G$ is important in determining how much the DW pinning is suppressed in comparison with a free DW $(G=0)$ which exhibits the largest value of the DW coercive field $H_{\mathrm{CW}}(0)$. As a consequence of this generality, the $H_{\mathrm{CW}}(G)$ dependence is likely to be responsible for such rather peculiar, not yet well described effects, like the $H_{\mathrm{CW}}$ dependence on domain structure subtle features or on the size and shape of the sample, experimentally observed on various materials. ${ }^{8,9}$

\section{MODEL}

\section{A. General considerations}

In our model calculations, we consider a certain part of a given sample, with just one rigid plane $180^{\circ} \mathrm{DW}$, characterized by its position coordinate, $x_{\mathrm{DW}}$. We neglect any effect related to the internal DW degrees of freedom (wall flexibility, internal deformation modes, etc.). In this sense, all the quantities introduced here are to be interpreted as averages over the whole DW surface. We assume that the DW sweeps the distance $2 l$ (with $-l \leqslant x_{\mathrm{DW}} \leqslant l$ ) when the sample is brought from saturation to saturation, which means that $2 l$ is of the order of the average magnetic domain size. Finally, we assume the problem to have a scalar nature, with all fields and magnetization vectors aligned along the same direction, parallel to the DW surface.

In order to calculate the DW coercive field, $H_{\mathrm{CW}}$, we have to evaluate the amount of energy dissipation during the DW motion. This can be done if we know the equation governing the DW dynamics. The simplest assumption, supported by many experiments on various materials ${ }^{10,11}$ is that the DW motion has a viscous character, and thus obeys an equation of the form 


$$
(G \tau) v_{\mathrm{DW}}=H_{a}(t)-H_{i}\left(x_{\mathrm{DW}}\right)=H_{a}(t)-G x_{\mathrm{DW}}-H_{p}\left(x_{\mathrm{DW}}\right)
$$

where $v_{\mathrm{DW}}=d x_{\mathrm{DW}} / d t$ is the DW velocity, $H_{a}(t)$ is the applied field, assumed to be a known function of time, and $H_{i}\left(x_{\mathrm{DW}}\right)$ is the DW position-restoring field discussed in the introduction [Eq. (1)]. The damping coefficient multiplying $v_{\text {DW }}$ in Eq. (2) is determined by the dissipation mechanism acting on the system. By expressing it as $(G \tau)$, we introduce the characteristic time constant $\tau$ of the process. In metallic materials, where the dissipation takes place dominantly through eddy currents, $(G \tau)=2 I_{s} \sigma \gamma d$, where $I_{s}$ is the saturation magnetization, $\sigma$ is the electric conductivity, $d$ is the sample thickness, and $\gamma$ is a numerical factor $(\gamma \approx 0.1356$ for $l \gg d$ (Ref. 12). In nonconducting systems, dissipation may be due to other mechanisms, like spin relaxation, and $(G \tau)$ generally attains much smaller values.

According to Eq. (2), the set of the DW rest positions $\left(v_{\mathrm{DW}}=0\right)$, which is described by the quasistatic magnetization curve of the system, obeys the equation

$$
H_{a}(t)-G x_{\mathrm{DW}}-H_{p}\left(x_{\mathrm{DW}}\right)=0 .
$$

Since, as discussed below, $H_{p}\left(x_{\mathrm{DW}}\right)$ values fluctuate around zero, the $x_{\mathrm{DW}}\left(H_{a}\right)$ dependence averaged over the stochastic fluctuations is simply $\left\langle x_{\mathrm{DW}}\right\rangle=H_{a} / G$. This result shows that the field gradient, $G$, is directly related to the slope of the magnetization curve, $\mu=I_{s} /(G l)$, averaged over the stochastic fluctuations. Any individual magnetization curve, however, will show irregular deviations from this average behavior, as a consequence of the random fluctuations of the pinning field $H_{p}\left(x_{\mathrm{DW}}\right) . H_{p}$ is proportional to the local gradient of the DW energy, $H_{p}\left(x_{\mathrm{DW}}\right)=\left[d \sigma_{w}\left(x_{\mathrm{DW}}\right) / d x_{\mathrm{DW}}\right] /$ $\left(2 I_{s}\right)$. Due to the nonuniformity of the sample (fluctuations of anisotropy, exchange, defects, etc.) $\sigma_{w}\left(x_{\mathrm{DW}}\right)$ fluctuates randomly around some constant average value $\left\langle\sigma_{w}\left(x_{\mathrm{DW}}\right)\right\rangle$. This means, that the DW cannot store energy during its motion, and consequently that the wall-pinning field $H_{p}\left(x_{\mathrm{DW}}\right)$ fluctuates around zero.

\section{B. Energy losses and magnetic hysteresis}

Given the viscouslike dynamics described by Eq. (2), where the DW velocity is proportional to the local magnetic field, there is always a finite dynamic width of the hysteresis loop, $H_{\text {dyn }}$, for a given finite rate of change of magnetization, even without any static DW $\operatorname{pinning}^{12}\left(H_{p}=0\right)$ :

$$
H_{\mathrm{dyn}}=\left\langle H_{a}-H_{i}\right\rangle=(G \tau)\left\langle v_{\mathrm{DW}}\right\rangle
$$

However, if the random pinning field, $H_{p}$, is also present, there is an additional contribution to dissipation, and a more refined analysis is needed. In this case, an estimate of the pertinent coercive field, $H_{\mathrm{DW}}$, can be obtained by comparing two different expressions for the mean energy loss during the DW motion. On one hand, we know that, given a DW hysteresis loop with the half-width, $H_{\mathrm{DW}}$, the mean rate $\langle d E / d t\rangle$ of the energy dissipation per unit time and unit DW area is

$$
\left\langle\frac{d E}{d t}\right\rangle=2 I_{s} H_{\mathrm{DW}}\left\langle v_{\mathrm{DW}}\right\rangle=2 I_{s} H_{\mathrm{DW}} \frac{d H_{a} / d t}{G} .
$$

Equation (5) is valid for constant rate of field sweep $d H_{a} / d t$. On the other hand, after taking into account that the energy associated with the term $G x_{\text {DW }}$ is reversibly stored and released and that the wall-pinning field $H_{p}$ is zero on the average taken over the stochastic fluctuations, we obtain from Eq. (2):

$$
\left\langle\frac{d E}{d t}\right\rangle=2 I_{s}(G \tau)\left\langle v_{\mathrm{DW}}^{2}\right\rangle .
$$

By comparing these two expressions for the energy dissipation rate, we obtain

$$
H_{\mathrm{DW}}=(G \tau) \frac{\left\langle v_{\mathrm{DW}}^{2}\right\rangle}{\left\langle v_{\mathrm{DW}}\right\rangle},
$$

which links the value of the coercive field $H_{\mathrm{DW}}$ to the stochastic character of the DW velocity $v_{\mathrm{DW}}$. The quantity $H_{\mathrm{CW}}$ previously introduced is just the quasistatic limit of $H_{\mathrm{DW}}$ :

$$
H_{\mathrm{CW}}=(G \tau) \lim _{\left\langle v_{\mathrm{DW}}\right\rangle \rightarrow 0} \frac{\left\langle v_{\mathrm{DW}}^{2}\right\rangle}{\left\langle v_{\mathrm{DW}}\right\rangle}
$$

In the numerical studies presented below, a small but nevertheless finite DW mean velocity must be used. In this case, the purely dynamic term (4) can give an additional contribution which alters the value of the DW coercive field $H_{\mathrm{CW}}$. To avoid this spurious effect, the pure dynamic part was always subtracted from the numerical estimates, i.e., $H_{\mathrm{CW}}$ was evaluated as

$$
H_{\mathrm{CW}} \approx(G \tau)\left(\frac{\left\langle v_{\mathrm{DW}}^{2}\right\rangle}{\left\langle v_{\mathrm{DW}}\right\rangle}-\left\langle v_{\mathrm{DW}}\right\rangle\right)
$$

for small DW velocity $v_{\mathrm{DW}}$.

\section{Stochastic pinning field, Wiener-Lévy process}

In the frame of the present description, the microstructural properties of a given material determine the statistical properties of the $H_{p}\left(x_{\mathrm{DW}}\right)$ stochastic process, and we have to make some explicit assumption on such properties before being able to make any quantitative prediction for the system. Investigations by several authors ${ }^{10,11}$ on picture-frame single crystals containing a very simple domain structure have shown that the statistical properties of the wall-pinning field $H_{p}$ are not far from those of a Wiener-Lévy (WL) process, i.e.,

$$
\frac{d H_{p}}{d x_{\mathrm{DW}}}=\frac{d W}{d x_{\mathrm{DW}}},
$$

where the WL process $W\left(x_{\mathrm{DW}}\right)$ is characterized by independent increments $d W$ obeying the equation

$$
\langle d W\rangle=0, \quad\left\langle|d W|^{2}\right\rangle=2\left(A_{H}^{2} / \xi_{2}\right) d x_{\mathrm{DW}},
$$


where $A_{H}$ is a parameter with the dimension of field that measures the WL process intensity and $\xi_{2}$ is a proper correlation length, used later in this section.

A WL process is self-similar on all scales, and this has two important, but rather unphysical consequences: (1) the process $W\left(x_{\mathrm{DW}}\right)$ is nonstationary in such a way that the wallpinning field $H_{p}$ can reach arbitrarily high values, (2) the process $W\left(x_{\mathrm{DW}}\right)$ is nondifferentiable at any point. The derivative $d W / d x_{\mathrm{DW}}$ used in (10) is actually only a formal notation to indicate a white noise process.

The fact that $W$ is nondifferentiable implies that the slope $d H_{p} / d x_{\text {DW }}$ cannot be defined no matter how small is the DW displacement in $x_{\mathrm{DW}}$. On the contrary, we would expect that, on the average, $d H_{p} / d x_{\mathrm{DW}} \propto\left(1 / \chi_{i}\right)$, where $\chi_{i}$ is the initial susceptibility. In order to overcome these drawbacks, we describe the stochastic behavior of the wall-pinning field $H_{p}\left(x_{\mathrm{DW}}\right)$ by including two finite correlation lengths, $\xi_{1}$ and $\xi_{2}\left(\xi_{1} \leqslant \xi_{2}\right)$, into the WL process. The physical meaning of the correlation lengths is following: (1) for $\Delta x_{\mathrm{DW}} \gg \xi_{2}$ the pinning field $H_{p}\left(x_{\mathrm{DW}}\right)$ becomes stationary and fluctuates within a finite range; (2) for $\Delta x_{\mathrm{DW}} \ll \xi_{1}$ the slope $d H_{p} / d x_{\mathrm{DW}}$ attains a well-defined value related to the local short-scale initial susceptibility of the DW at that position. The short-scale susceptibility is given by local properties of the wall-pinning field $H_{p}$ and it is in general very different from the large-scale susceptibility, $\propto 1 / G$, corresponding to the large-scale slope of the hysteresis loop.

These features are described by the set of coupled Langevin equations

$$
\frac{d H_{p}}{d x_{\mathrm{DW}}}+\frac{H_{p}}{\xi_{2}}=S\left(x_{\mathrm{DW}}\right), \quad \frac{d S}{d x_{\mathrm{DW}}}+\frac{S}{\xi_{1}}=\frac{1}{\xi_{1}} \frac{d W}{d x_{\mathrm{DW}}},
$$

where the WL process $W\left(x_{\mathrm{DW}}\right)$ is characterized by Eq. (11) and $S\left(x_{\mathrm{DW}}\right)$ describes the slope of $H_{p}$.

It should be noted that Eq. (12) can be rewritten as

$$
\frac{d W}{d x_{\mathrm{DW}}}=\frac{H_{p}}{\xi_{2}}+\left(1+\frac{\xi_{1}}{\xi_{2}}\right) \frac{d H_{p}}{d x_{\mathrm{DW}}}+\xi_{1} \frac{d^{2} H_{p}}{d x_{\mathrm{DW}}^{2}},
$$

which gives in the limit of lengths $\xi_{1} \rightarrow 0$ and $\xi_{2} \rightarrow \infty$ just Eq. (10).

\section{Dimensionless description}

We can introduce a convenient dimensionless description by normalizing the relevant quantities with respect to the distance $\xi_{2}$, time $\tau$, field $A_{H}$, and gradient $A_{H} / \xi_{2}$ in the following way: DW position $x=x_{\mathrm{DW}} / \xi_{2}$, time $u=t / \tau$, DW velocity $v=d x / d u=\tau v_{\mathrm{DW}} / \xi_{2}$, magnetic fields $h_{a}(u)$ $=H_{a}(t) / A_{H}, \quad h_{p}(x)=H_{p}\left(x_{\mathrm{DW}}\right) / A_{H}, \quad$ coercive field $h_{\mathrm{CW}}$ $=H_{\mathrm{CW}} / A_{H}, \quad$ gradient $\quad \beta=G \xi_{2} / A_{H}, \quad$ slope $s(x)=S\left(x_{\mathrm{DW}}\right) \xi_{2} / A_{H}$. The correlation lengths are characterized by their ratio $\alpha=\xi_{1} / \xi_{2}$, while the dimensionless WL process is $w=W / A_{H}$.

In terms of these quantities, the basic equation (2) takes the form

$$
\beta v=h_{a}(u)-\beta x-h_{p}(x) .
$$

Equations (12) and (11) become

$$
\begin{gathered}
\frac{d h_{p}}{d x}+h_{p}=s(x), \quad \frac{d s}{d x}+\frac{s}{\alpha}=\frac{1}{\alpha} \frac{d w}{d x}, \\
\langle d w\rangle=0, \quad\left\langle|d w|^{2}\right\rangle=2 d x .
\end{gathered}
$$

The dimensionless coercive field, $h_{\mathrm{CW}}$, can be expressed similarly to Eqs. (8) and (9) as

$$
h_{\mathrm{CW}}=\beta \lim _{\langle v\rangle \rightarrow 0} \frac{\left\langle v^{2}\right\rangle}{\langle v\rangle}, \quad h_{\mathrm{CW}} \approx \beta\left(\frac{\left\langle v^{2}\right\rangle}{\langle v\rangle}-\langle v\rangle\right),
$$

where the equation to the right is valid for small DW velocity $v$. The evaluation of the coercive field $h_{\mathrm{CW}}$ is thus reduced to the problem of the evaluation of $\left\langle v^{2}\right\rangle$ and $\langle v\rangle$.

\section{E. Pure WL process}

As a first step, we calculate the DW coercive field for a stochastic pinning field, $H_{p}$, following the pure WL process [Eqs. (10) and (11)], i.e., for correlation lengths $\xi_{1} \rightarrow 0$ and $\xi_{2} \rightarrow \infty$, which implies $\alpha \rightarrow 0$. In such a simple case, when $\alpha(d s / d x)$ is negligible in comparison to $d w / d x$, so that $\left(d h_{p} / d x\right)+h_{p}=(d w / d x)$ according to Eq. (15). In addition, since the correlation length $\xi_{2} \rightarrow \infty$, we have to consider DW displacements $\Delta x \ll 1$, which implies that the wall-pinning field is given just by the WL stochastic process, $h_{p}(x) \approx w(x)$. In this case, by taking the time derivative of Eq. (14), we obtain

$$
\frac{d v}{d u}+(v-\langle v\rangle)=\frac{1}{\beta} \frac{d w}{d u}, \quad\left\langle|d w|^{2}\right\rangle=2 d x=2 v d u
$$

The stationary probability density $P_{0}$ for the values of the DW velocity $v$ at a generic time $u$ is obtained by solving the stationary Fokker-Planck equation associated with Eq. (18):

$$
\left[(v-\langle v\rangle) P_{0}\right]+\frac{1}{\beta^{2}} \frac{d\left[v P_{0}\right]}{d v}=0,
$$

which gives

$$
P_{0}(v) \propto v^{c-1} e^{-\beta^{2} v}, \quad c=\beta^{2}\langle v\rangle .
$$

In order to calculate the DW coercive field $h_{\mathrm{CW}}$, we need to evaluate $\left\langle v^{2}\right\rangle$ from the probability distribution $P_{0}$ :

$$
\left\langle v^{2}\right\rangle=\int_{0}^{\infty} v^{2} P_{0}(v) d v=\langle v\rangle^{2}+\frac{\langle v\rangle}{\beta^{2}}=\frac{1}{\beta^{4}}\left(c^{2}+c\right) .
$$

By substituting Eq. (21) into Eq. (17) we obtain the DW coercive field

$$
h_{\mathrm{CW}}=\frac{1}{\beta}, \quad H_{\mathrm{CW}}=\frac{A_{H}^{2} / \xi_{2}}{G} .
$$

It should be noted that this result is meaningful if the parameter of the WL process $A_{H}$ is chosen in such a way that the ratio $A_{H}^{2} / \xi_{2}$ remains finite for the correlation length $\xi_{2} \rightarrow \infty$. 


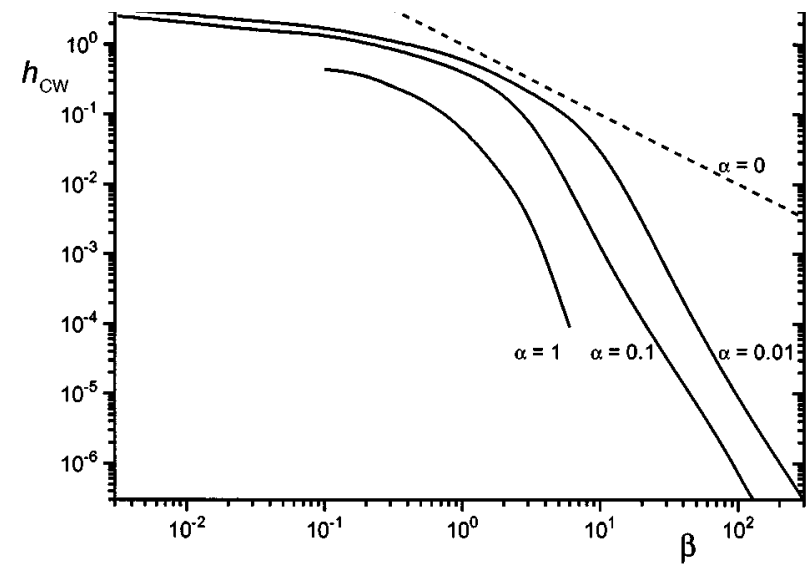

FIG. 2. The dimensionless domain-wall coercive field, $h_{\mathrm{CW}}$, calculated numerically for finite correlation lengths, $\xi_{1}$ and $\xi_{2}$, for constant ratio $\alpha=\xi_{1} / \xi_{2}=1,0.1$, and 0.01 for a wide range of values of the dimensionless field gradient $\beta$ along with the $h_{\mathrm{CW}}$ $\propto 1 / G$ dependence evaluated according to Eq. (22) for the pure WL process (i.e., for $\alpha=0$ ). Asymptotic behavior for very small and very large $\beta$ values (in the range not accessible by experiments) is also clearly seen in this plot.

Equation (22) gives a hyperbolic decrease of the DW coercive field $H_{\mathrm{CW}}$ with increasing magnetic field gradient, $G$, independent of $\left\langle v_{\mathrm{DW}}\right\rangle$. The divergence in $H_{\mathrm{CW}}$ for zero gradient, $G$, is a consequence of the absence of any limit on the correlation length $\xi_{2}$. A more realistic situation is obtained when finite correlation lengths $\xi_{2}$ and $\xi_{1}$ (i.e., finite $\left.\alpha=\xi_{1} / \xi_{2}\right)$ are considered, as discussed in the following subsection.

\section{F. Numerical solution for finite $\alpha$}

No simple analytic results could be obtained for finite $\alpha$, so that this case was studied by computer simulations. A proper statistical ensemble of pinning field profiles was generated through Eqs. (15) and (16). On the basis of these profiles, the behavior of the DW velocity $v$ was numerically generated from Eq. (14) and the DW coercive field $h_{\mathrm{CW}}$ was estimated by Eq. (17). Calculations were performed as functions of the field gradient $\beta$, for three different values of $\alpha=0.01,0.1$, and 1 . All the simulations were performed under sufficiently small mean dimensionless DW velocity $\langle v\rangle$, in order to avoid spurious dynamic effects. According to Eq. (20), the quasistatic regime is attained when $c=\beta^{2}\langle v\rangle \ll 1$. All calculations were performed at $c=0.1$. In addition, the DW coercive field $h_{\mathrm{CW}}$ was evaluated from the approximate expression given in Eq. (17), in order to correct for any residual dynamic effect.

Results of these numerical simulations for $\alpha=0.01,0.1$, and 1 are plotted in Fig. 2. The dependence of the DW coercive field $h_{\mathrm{CW}}=1 / \beta$, corresponding to the pure WL process $(\alpha=0)$ is also given. For finite $\alpha$ values where the statistics of the pinning field is considerably affected, the results clearly show a large departure of $h_{\mathrm{CW}}$ from the $1 / \beta$ dependence. In the low-gradient region $(\beta \ll 1)$, the $h_{\mathrm{CW}}$ dependence on the field gradient $\beta$ is much weaker than the $1 / \beta$ function, while in the opposite limit of very high gradients, $\beta$, the coercive field, $h_{\mathrm{CW}}$, decreases approximately

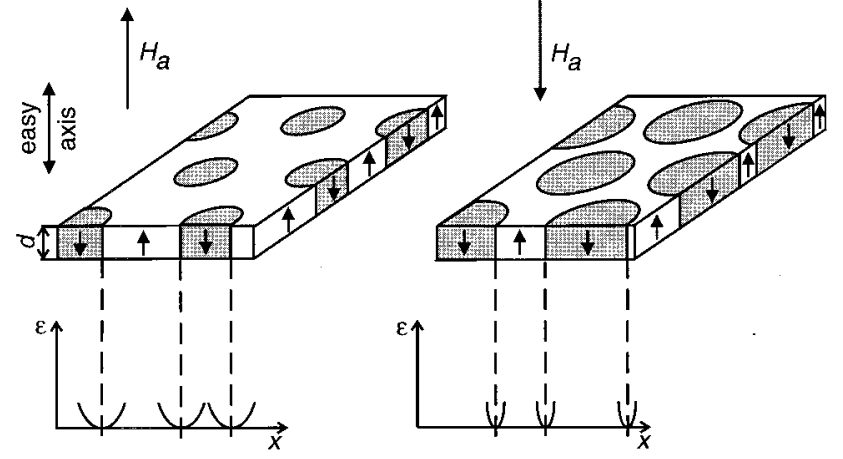

FIG. 3. Schematic plot of a hexagonal array of bubble domains in a thin film with large uniaxial magnetic anisotropy perpendicular to the sample plane. The bias field, $H_{a}$, applied parallel to the magnetization vector inside bubbles makes the bubbles large, and pushes DW's close to one another. Consequently, the position of DW's is more restricted by the domain structure energy, $\varepsilon$, i.e., the local field gradient, $G$, experienced by DW's is large and the measured DW coercive field $H_{\mathrm{CW}}$ is low. Under the $H_{a}$ antiparallel to the bubble magnetization, the bubbles are small and the DW's are far from one another. In this case the field gradient $G$ is low and $H_{\mathrm{CW}}$ is high.

exponentially with the field gradient $\beta$, i.e. much more strongly than $1 / \beta$. These numerical curves are the basis for the interpretation of the experimental results presented in the following section.

\section{EXPERIMENT}

DW hysteresis in samples of magnetic garnet films with chemical composition ( $\mathrm{YSmCa})_{3}(\mathrm{FeGe})_{5} \mathrm{O}_{12}$ (sample no. 1) and $(\mathrm{YSmCaLu})_{3}(\mathrm{FeGe})_{5} \mathrm{O}_{12}$ (sample no. 2) was measured and the results compared with the above model.

Thin film samples of thickness, $d$, were grown by the liquid phase epitaxy (LPE) method on (111) gadolinium gallium garnet (GGG) substrates. Due to the growing conditions of the preparation process they exhibited large uniaxial anisotropy, $K_{u}$, with the easy axis of magnetization perpendicular to the film plane. The original cubic crystalline anisotropy of the garnet material was negligibly small with respect to the induced uniaxial one. The saturation magnetization, $I_{s}$, inside all domains was perpendicular to the film plane and the metastable close-packed bubble domain structure of the type shown in Fig. 3 was produced by magnetizing the samples up to the saturation in their planes and by a subsequent slow decrease of the applied field down to zero.

The basic parameters of the samples chosen for the hysteresis experiments were determined at room temperature by standard methods for bubble garnet film characterization ${ }^{13}$ and they are listed in Table I.

The low-frequency $(200 \mathrm{~Hz})$ domain-wall oscillation method based on the response of the domain system to a small ac magnetic field perpendicular to the film plane ${ }^{14}$ was used to determine the domain wall coercive field, $H_{\mathrm{CW}}$, of the samples. The field, $H_{\mathrm{CW}}$, was measured in each sample on a series of the bubble domain structures whose geometrical parameters were changed step by step by an externally applied static bias field, $H_{a}$, normal to the sample plane, see 
TABLE I. Parameters of the thin film samples: thickness $d$, saturation magnetization $I_{s}$, uniaxial anisotropy constant $K_{u}$, characteristic material length $\lambda$, normalized to the sample thickness $d$, and the domain-wall width parameter $\delta_{w}=\sqrt{A / K_{u}}$, where $A \approx 2.05 \times 10^{-12} \mathrm{~J} / \mathrm{m}$ is the exchange constant of garnets.

\begin{tabular}{cccccc}
\hline \hline $\begin{array}{c}\text { Sample } \\
\text { No. }\end{array}$ & $\begin{array}{c}d \\
(\mu \mathrm{m})\end{array}$ & $\begin{array}{c}I_{s} \\
(\mathrm{mT})\end{array}$ & $\begin{array}{c}K_{u} \\
\left(\mathrm{~J} / \mathrm{m}^{3}\right)\end{array}$ & $\begin{array}{c}\lambda \\
(1)\end{array}$ & $\begin{array}{c}\delta_{w} \\
(\mathrm{~nm})\end{array}$ \\
\hline 1 & 5.3 & 20.0 & 634 & 0.09 & 57 \\
2 & 2.8 & 23.8 & 1510 & 0.30 & 37 \\
\hline \hline
\end{tabular}

Fig. 3. A typical result of the DW coercive field $H_{\mathrm{CW}}\left(H_{a}\right)$ measurement is shown in Fig. 4. While density of bubble magnetic domains is constant, size of particular domains changes significantly by application of bias magnetic field $H_{a}$ as indicated by insets in Fig. 4. While for small domains with DW's far from each other the gradient $G$ of the effective local DW position-restoring magnetic field is small and consequently the coercive field $H_{\mathrm{CW}}$ is large (right side of Fig. 4), in opposite bias field the DW's of bubble domains are close to each other, $G$ is large, and $H_{\mathrm{CW}}$ is small (left side of Fig. 4).

Using the magnetic parameters of the samples and the geometrical parameters of their domain structures at different bias field values, $H_{a}$, the effective local field gradients, $G=G\left(H_{a}\right)$, were computed according to the formulas given in Ref. 5. In dimensionless variables, the measured DW coercive field, $H_{\mathrm{CW}}$, was normalized to the parameter of the WL process $A_{H}$ and the gradient, $G$, computed in this way was normalized to $A_{H} / \xi_{2}$. The normalization parameters $A_{H}$ and $A_{H} / \xi_{2}$ were used as variable fitting parameters to fit

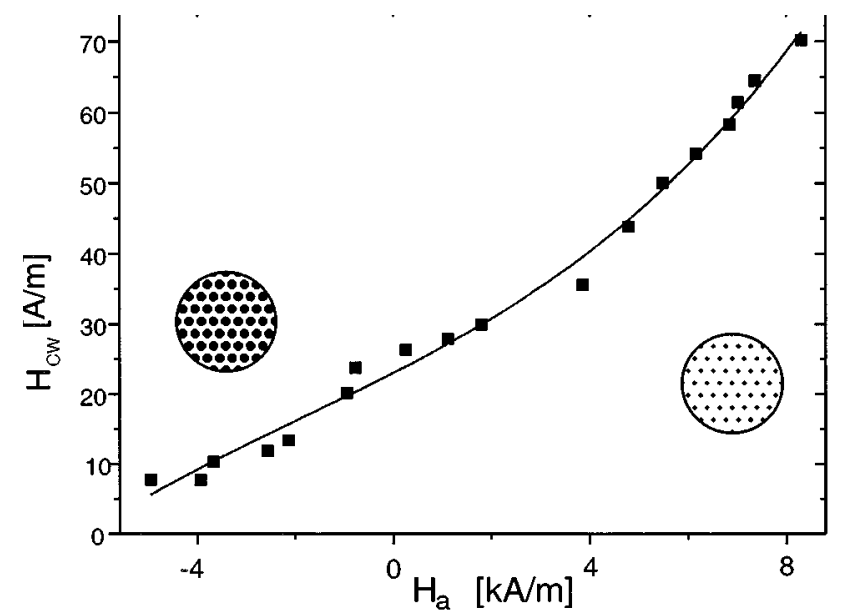

FIG. 4. The dependence of the DW coercive field, $H_{\mathrm{CW}}$, measured on a hexagonal array of bubble domains in sample no. 1 at various bias field, $H_{a}$, parallel (negative, left) and antiparallel (positive, right) to the magnetization vector inside the bubbles. Computation of the eye-leading curve was based on a best fit thirdorder polynom. Changes in domain structure (compare to Fig. 3) are illustrated in two circular insets corresponding to large positive and negative applied field $H_{a}$, respectively: The period of the domain structure keeps constant but the DW positions change (the bubble domain diameter varies) from one experimental point to another. $H_{\mathrm{CW}}$ changes considerably with changing distance between neighboring DW's.

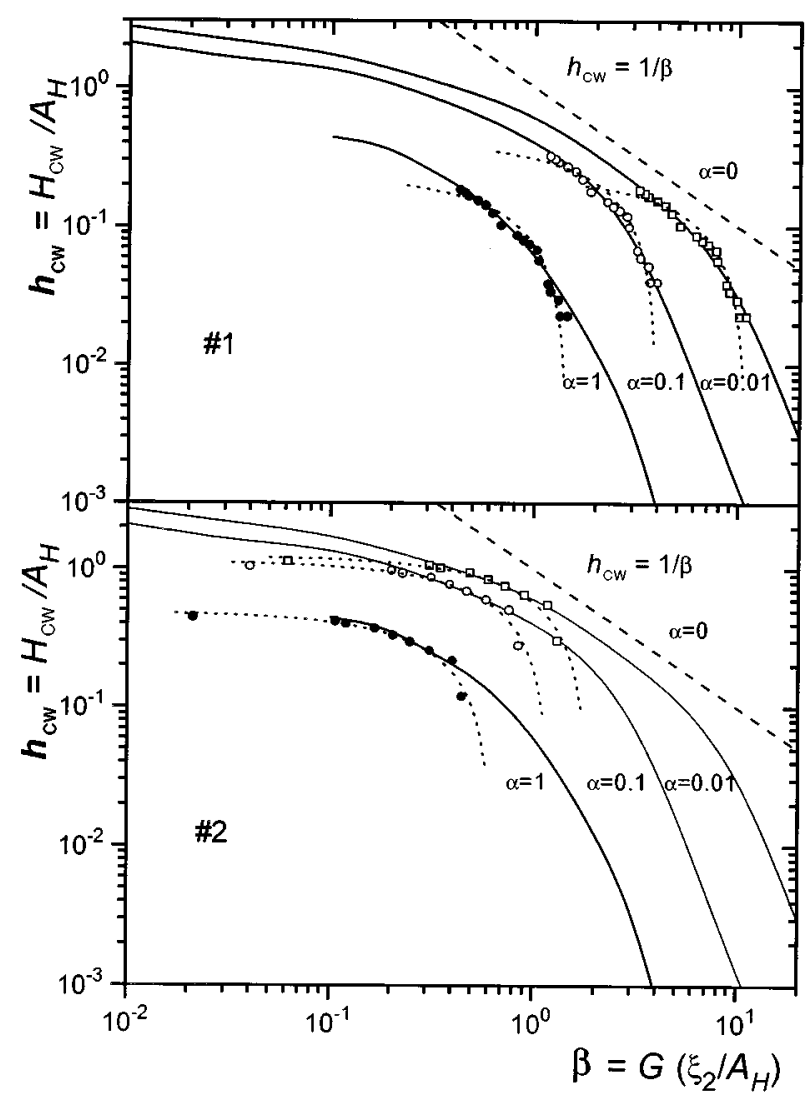

FIG. 5. Experimental dependence of the measured DW coercive field, $h_{\mathrm{CW}}$, on the local magnetic field gradient $\beta$, evaluated from domain structure modifications (see Fig. 4) of sample nos. 1 and 2. The experimental data were fitted independently to the three curves calculated for ratios of the correlation lengths $\alpha=\xi_{1} / \xi_{2}=1$ (full circles), 0.1 (open circles), and 0.01 (squares). The values of the normalization parameters were used as the fitting parameters: $h_{\mathrm{CW}}=H_{\mathrm{CW}} / A_{H}$ and $\beta=G /\left(A_{H} / \xi_{2}\right)$ (see Table II). The quality of fit is very comparable for all three values of $\alpha$ and so it is not possible to determine in this way the best $\alpha$ value. However, we believe that the value $\alpha=1$ is probably the closest to the real situation. The dashed line corresponds to the unrealistic pure WL process $(\alpha=0)$. The fits of the theoretical linear approximation discussed in Ref. 5 are shown as the dotted curves for each of the samples.

the dependence of the dimensionless experimental coercive field, $h_{\mathrm{CW}}=H_{\mathrm{CW}} / A_{H}$, on the dimensionless field gradient, $\beta=G \xi_{2} / A_{H}$, to the computed dimensionless $h_{\mathrm{CW}}(\beta)$ dependencies. The experimental data of both samples were fitted to the $h_{\mathrm{CW}}(\beta)$ curves, which were calculated independently for the three values of the ratio of the correlation lengths $\alpha=\xi_{1} / \xi_{2}=1,0.1$ and 0.01 . The result is summarized in Fig. 5 where all computed dimensionless $h_{\mathrm{CW}}(\beta)$ dependencies are plotted along with the fitted experimental data. Values of the fitting parameters are in Table II.

\section{DISCUSSION}

\section{A. Domain structures}

Generally, there are several different contributions to the quasistatic hysteresis losses which occur during any change of the magnetization state. While the contribution due to sud- 
TABLE II. The parameter $A_{H}$ of the WL process and the correlation lengths $\xi_{1}$ and $\xi_{2}$ of the pinning field obtained from the fit of the experimental data to the curves calculated for three different assumed values of $\alpha=\xi_{1} / \xi_{2}=1,0.1$, and 0.01 (see Fig. 5). The value of $A_{H}^{2} / \xi_{2}$ characterizing the WL process is also presented.

\begin{tabular}{clcccc}
\hline \hline $\begin{array}{c}\text { Sample } \\
\text { No. }\end{array}$ & $\begin{array}{c}\alpha \\
(1)\end{array}$ & $\begin{array}{c}A_{H} \\
(\mathrm{~A} / \mathrm{m})\end{array}$ & $\begin{array}{c}\xi_{1} \\
(\mathrm{~nm})\end{array}$ & $\begin{array}{c}\xi_{2} \\
(\mathrm{~nm})\end{array}$ & $\begin{array}{c}A_{H}^{2} / \xi_{2} \\
\left(10^{11} \mathrm{~A}^{2} / \mathrm{m}^{3}\right)\end{array}$ \\
\hline 1 & 1 & 350 & 64 & 64 & 19 \\
1 & 0.1 & 200 & 10 & 100 & 4 \\
1 & 0.01 & 350 & 4.8 & 480 & 2.6 \\
2 & 1 & 140 & 7.4 & 7.4 & 27 \\
2 & 0.1 & 60 & 0.6 & 6 & 6 \\
2 & 0.01 & 55 & 0.085 & 8.5 & 3.6 \\
\hline \hline
\end{tabular}

den flips over of the magnetization is mostly linked with extreme material parameters and/or with extreme geometry of the material grains, and while the contribution due to rotation of the magnetization vector inside magnetic domains or grains is generally negligible, the recorded quasistatic hysteresis losses are usually caused by generation and/or annihilation of magnetic domains and domain walls and by translation of the already existing domain walls through the nonuniform material. We study the latter process in this paper, namely the pinning and depinning of domain walls in soft magnetic materials. In order to investigate the coercive fields caused by the pure DW pinning and depinning only, we keep such experimental conditions and the DW geometry, where contributions of any DW generation and annihilation, and of any rotation of the magnetization vector can be neglected.

The necessary condition for experimental verification which describes the presented theoretical model of the relationship between the efficiency of pinning of the domain walls by the sample random defects (i.e., the value of the DW coercive field, $H_{\mathrm{CW}}$ ) and the magnitude of the largescale position stiffness of the domain walls within the domain structure of the sample (described by the effective local field gradient $G$ ), is the requirement of uniformity of the type, of the quality and of the identical local conditions of all the domain walls involved. In other words it means that the investigated domain structure in the sample should be regular and periodic. Such domain structures not only insure obtaining equivalent response to identical applied field excitation of any of the walls but they also make it possible to determine the total area of the walls, to express the total energy of the domain structure in a reasonable analytical shape, and to normalize the calculated values to unit DW area. Uniaxial plane-parallel magnetic garnet films prepared by liquid phase epitaxy support domain structures (e.g., a system of parallel stripe domains magnetized alternatively "up"' and "down', perpendicularly to the sample plane, or a close-packed array of cylindrical bubble domains, see Fig. 2) with the required regularity, periodicity and the mutual equivalence of all the domain walls. The range of the local field gradients at the DW positions reached by field modifications of the bubble domain structures is considerably wider (by about one order of magnitude) than the range of the field gradient $G$ reached by similar modifications of the stripe domains. ${ }^{5}$ Therefore, samples of the magnetic garnet films with the cylindrical bubble domain structure were used in our experiment and the relevant formulas from ${ }^{5}$ were used for determination of the magnitude of the field gradient $G$ in bubble domain arrays with different diameters of bubbles. An external uniform magnetic field, $H_{a}$, along the easy axis of magnetization of the samples changed the bubble diameter easily, see Fig. 3. By application of $H_{a}$, values of the field gradient $G$ were varied within the ratio of about 1:5.

\section{B. Effective local field gradients}

It was shown in Ref. 5 that pressures acting on the domain walls in any domain structure originate from the shape of the domain structure energy, $\varepsilon$. They can be understood as being due to presence of nonuniform effective internal fields, $H_{i}\left(x_{\mathrm{DW}}\right) \sim\left(\partial \varepsilon / \partial x_{\mathrm{DW}}\right)$, and the shape of $H_{i}\left(x_{\mathrm{DW}}\right)$ close to any equilibrium DW position can be described (up to a linear approximation) with the aid of effective field gradients at the wall sites, $H_{i}(x) \approx G x_{\mathrm{DW}}$. This approximation was subjected to the statistical analysis of the present paper. The analysis came to the conclusion that the larger is the field gradient $G$, the more effectively it decreases the sensitivity of the DW to feel presence of the wall pinning defects. This drop of the wall sensitivity brings about a decrease of the sample DW coercive field and of the static hysteresis losses. It is important to emphasize at this point that the effective field gradient, $G \sim\left(\partial^{2} \varepsilon / \partial x_{\mathrm{DW}}^{2}\right)$, is composed of at least three effective field gradient contributions, expressed as the second derivatives of the demagnetizing energy, of the domain-wall energy, and of the Zeeman energy, together, and it is not identical with the real demagnetizing field gradients existing at the sites of the walls. The real demagnetizing field gradients existing at the DW positions are only one component of the total $G$ value.

The effective local magnetic field gradients, $G$, can be changed and controlled in various ways. In case of a single domain wall a straightforward application of an external magnetic gradient field can be used. ${ }^{7}$ In periodic domain structures, changes of dimensions of domains by application of an external uniform field, $H_{a}$, is the easiest way of influencing the field gradient $G$. Variation of dimensions of the sample presents another way of changing $G{ }^{9}$

In the present model, energy dissipation and coercivity are derived from the existence of DW Barkhausen jumps. These jumps are due to the fluctuating character of the internal DW position-restoring field, $H_{i}\left(x_{\mathrm{DW}}\right)=G x_{\mathrm{DW}}$ $+H_{p}\left(x_{\mathrm{DW}}\right)$. A local position of the DW expressed by Eq. (3) is stable if the slope $\left(G+d H_{p} / d x_{\mathrm{DW}}\right)_{x_{\mathrm{DW}}}>0$, and it is unstable if $\left(G+d H_{p} / d x_{\mathrm{DW}}\right)_{x_{\mathrm{DW}}}<0$. When the DW reaches a local maximum, $\left(G+d H_{p} / d x_{\mathrm{DW}}\right)_{x_{\mathrm{DW}}}=0$, it irreversibly jumps to a new stable position and dissipates some energy. The pinning field $H_{p}\left(x_{\mathrm{DW}}\right)$ has an equal proportion of regions where $d H_{p} / d x_{\mathrm{DW}}$ is positive and negative, but this proportion is altered by the presence of the term $G x_{\mathrm{DW}}$, which favors local positive slopes. With increasing $G$ the system progressively becomes more stable, the average size of Barkhausen jumps becomes smaller, and the associated energy dissipation is reduced. This is the basic mechanism responsible for the decrease of the DW coercive field $H_{\mathrm{CW}}$ with the field gradient $G$. In the case when $G$ is so large that 
it compensates any local negative slope in $H_{p}\left(x_{\mathrm{DW}}\right)$, we get only positive slopes and the DW motion becomes fully reversible $\left(H_{\mathrm{CW}}=0\right)$. See the schematic illustration in the bottom plots of Fig. 1.

To analyze the coercive pinning of DW under different conditions in detail, we introduced three coercive fields defined in the following way: (i) $H_{\mathrm{DW}}$ is the half-width of the total hysteresis loop at finite DW velocity $v_{\mathrm{DW}}$, (ii) $H_{\mathrm{dyn}}$ is the half-width of the hysteresis loop caused only by the dynamic losses, and (iii) the DW coercive field $H_{\mathrm{CW}}$ is the static DW pinning coercive field for $v_{\mathrm{DW}}$ approaching zero $H_{\mathrm{CW}}=\lim _{\left\langle v_{\mathrm{DW}}\right\rangle \rightarrow 0}\left(H_{\mathrm{DW}}-H_{\mathrm{dyn}}\right)$. [It should be noted that the static DW pinning coercive field, $H_{\mathrm{CW}}$, can be expressed also as $H_{\mathrm{CW}}=\lim _{\left\langle v_{\mathrm{DW}}\right\rangle \rightarrow 0} H_{\mathrm{DW}}$ because $\lim _{\left\langle v_{\mathrm{DW}}\right\rangle \rightarrow 0} H_{\mathrm{dyn}}=0$, but as the expression $\left(H_{\mathrm{DW}}-H_{\mathrm{dyn}}\right)$ is much less velocitydependent than $H_{\mathrm{DW}}$, the former expression is more suitable for physical considerations and numerical calculations.]

\section{The theory and the experimental fit}

While it is difficult to change the field gradient $\beta$ experimentally by more than 1 order of magnitude, the dimensionless coercive field can be calculated for a very wide range of field gradients. The general case of the coercive interaction of DW with the pinning field given by the WL process modified by two finite correlation lengths, $\xi_{1}$ and $\xi_{2}$, was investigated by a numerical simulation for the dimensionless gradient field, $\beta$, changing by more than 4 orders of magnitude (see Fig. 2). Such dependencies have a very specific shape. While a simple but unrealistic hyperbolic dependence of the dimensionless coercive field (the dashed straight line in Fig. 2 ) was obtained for the pinning field corresponding to the pure Wiener-Lévy process $(\alpha \rightarrow 0)$, the $h_{\mathrm{CW}}(\beta)$ dependence calculated for any finite ratio of the correlation lengths, $\alpha$, was close to the $1 / \beta$ hyperbolic dependence between about $\beta=1$ and $\beta=\alpha^{-1 / 2}$ only. The $h_{\mathrm{CW}}(\beta)$ dependencies calculated for different finite values of $\alpha$ are to a large extent similar to one another. The slope of the high-gradient tails for $\alpha=0.1$ and 0.01 can be approximated by $h_{\mathrm{CW}} \propto \beta^{-3.3}$ in the range $\beta \ll \alpha^{-1}$, and they are shifted with respect to each other by a factor 2.3 on the $\beta$ axis. In the low gradient range $(0.001<\beta<0.1)$ the coercive field varies with $\beta$ only slightly, with a slope of $h_{\mathrm{CW}} \propto \beta^{-0.2}$. In other words, the DW coercive field $H_{\mathrm{CW}}$ tends to a finite value, $H_{\mathrm{CW}}(0)$ as the field gradient $G$ approaches zero. Another realistic deviation from the hyperbolic pure WL dependence takes place for $G \gg A_{H} / \sqrt{\xi_{1} \xi_{2}}$, with the DW coercive field $H_{\mathrm{CW}}$ rapidly approaching zero. These results are in good agreement with the experimental findings and permit a quantitative description of the measured $H_{\mathrm{CW}}$ vs $G$ behavior.

This picture also makes the role of the correlation lengths $\xi_{1}$ and $\xi_{2}$ more transparent. According to the theory of Langevin equations, ${ }^{15}$ the process $S\left(x_{\mathrm{DW}}\right)$ of Eq. (12) takes values whose mean square is of the order of the correlation length $\xi_{1}$ times the intensity of the white noise term, and it controls the slope $d H_{p} / d x$, so that $d H_{p} / d x_{\mathrm{DW}} \sim A_{H} /$ $\sqrt{\xi_{1} \xi_{2}}$. This means that when the field gradient $G \gg A_{H} / \sqrt{\xi_{1} \xi_{2}}$, i.e., $\beta \gg \alpha^{-1 / 2}$, the process tends to become fully reversible and the DW coercive field $H_{\mathrm{CW}}$ quickly decays to zero. This explains the behavior of the DW coercive field $H_{\mathrm{CW}}$ for large $G$ values in Fig. 2. On the other hand, when the field gradient $G$ is low, the DW coercive field $H_{\mathrm{CW}}$ is controlled by the local maxims of the wall-pinning field $H_{p}\left(x_{\mathrm{DW}}\right)$ only. These can be estimated from Eq. (12), by considering the limit $\xi_{1} \rightarrow 0$ (i.e., by neglecting the fine slope details which are not important in determining the $H_{p}$ maxims). Then, the equation for the wall-pinning field $H_{p}$ reduces to the standard Langevin equation and, by considerations similar to the previous ones on the slope $S\left(x_{\mathrm{DW}}\right)$, we get the estimate $H_{p} \sim A_{H}$, independent of the field gradient $G$ and of ratio $\alpha=\xi_{1} / \xi_{2}$. This explains the low $G$ part of Fig. 2.

The numerical simulation according to Sec. II $\mathrm{F}$ enables calculation of dimensionless coercive field, $h_{\mathrm{CW}}$, as a function of the dimensionless field gradient, $\beta$, for various $\alpha$. Shape of the stochastic pinning field, $H_{p}$, is determined by three parameters: $A_{H}, \xi_{1}$, and $\xi_{2}$, which can be determined in general by fitting of the experimental $H_{\mathrm{CW}}(G)$ dependencies to the calculated $h_{\mathrm{CW}}(\beta)$ curves using the normalization parameters of the field, $A_{H}$, and of the field gradient, $A_{H} / \xi_{2}$, as the fitting parameters. Both the parameter of the WL process $A_{H}$ and $\xi_{2}$ can be determined in this way. However, the dimensionless $h_{\mathrm{CW}}(\beta)$ curves calculated for different $\alpha=\xi_{1} / \xi_{2}$ have similar shape (they are mostly just shifted with respect to each other). The quality of the fit is therefore nearly the same in all cases and we have to assume a particular value of $\alpha$ to determine also $\xi_{1}$. Moreover, the dependence of the DW coercive field $H_{\mathrm{CW}}(G)$ was measured in much narrower range of the experimental values of the field gradient $G$ (up to one order of magnitude) than the calculated $h_{\mathrm{CW}}(\beta)$, where the range of $\beta$ covered more than 4 orders of magnitude. Therefore, in Fig. 5 and Table II we present the fits of experimental data to the curves calculated for all the three investigated finite values of $\alpha=1,0.1$, and 0.01 . By choosing a particular value of $\alpha=\xi_{1} / \xi_{2}$ we determine the normalization parameters $A_{H}$ and $\xi_{2} / A_{H}$, the field $h_{\mathrm{CW}}=H_{\mathrm{CW}} / A_{H}$, the gradient $\beta=G \xi_{2} / A_{H}$ and $\xi_{1}=\alpha \xi_{2}$ from the fit. Their values for both samples are listed in Table II.

Considering the limited range of the experimental data, their scatter, and the smooth shape of the calculated curves, there can be easily an error of about $50 \%$ in determination of the parameter of the WL process $A_{H}, \xi_{1}$, and $\xi_{2}$ from the fit. The data of sample no. 1 fit the calculated curves in general better than those measured on sample no. 2. The normalization parameter of the magnetic field, $A_{H}$, was found to fall between 200 and $350 \mathrm{~A} / \mathrm{m}$ for sample no. 1 and between 55 and $140 \mathrm{~A} / \mathrm{m}$ for sample no. 2, for different $\alpha$. The shape of the pinning field is given by the Wiener-Lévy stochastic process according to Eq. (11) as $\left\langle|d W|^{2}\right\rangle=2\left(A_{H}^{2} / \xi_{2}\right) d x_{\mathrm{DW}}$. The value of $A_{H}^{2} / \xi_{2}$ was found for both samples between 2.6 and $27 \times 10^{11} \mathrm{~A}^{2} / \mathrm{m}^{3}$. The values of the correlation lengths of sample no. 1 are larger by about 1 order of magnitude than for sample no. 2.

The correlation lengths $\xi_{1}$ and $\xi_{2}$ were formally introduced to be distances such that for $\Delta x_{\mathrm{DW}} \ll \xi_{1}$ the slope, $d H_{p} / d x_{\mathrm{DW}}$, attained a well defined value and for $\Delta x_{\mathrm{DW}} \gg \xi_{2}$ the pinning field $H_{p}\left(x_{\mathrm{DW}}\right)$ becomes stationary. Qualitatively speaking it means that $\xi_{1}$ characterizes the finest profile of the pinning field experienced by DW, some- 
thing like an effective distance between relevant neighboring pinning points, whereas $\xi_{2}$ characterizes a critical distance the wall has to be shifted in order to "forget" past local pinning influences. Obviously, both such limits should be related to the DW width: the DW cannot effectively interact with any pinning field profile which is much finer than the DW width, and any shift of the DW by a distance, $\Delta x_{\mathrm{DW}}$, much larger than the DW width makes the DW forget its history surely. Such considerations focus the experimentally most relevant choice of $\alpha$ to $\alpha=1$, i.e., to the choice of a single correlation length $\xi_{1}=\xi_{2}=\xi$, with the expectation that $\xi$ will be of the order of the DW width parameter, $\delta_{w}=\sqrt{A / K_{u}}$, where $A \approx 2.05 \times 10^{-12} \mathrm{~J} / \mathrm{m}$ is the exchange constant of garnets. The results of such a choice give $\xi=64$ $\mathrm{nm}, \delta_{w}=57 \mathrm{~nm}$ for sample no. 1 and $\xi=7.4 \mathrm{~nm}, \delta_{w}=37$ $\mathrm{nm}$ for sample no. 2 . It is certainly a good agreement considering that the values of $\xi_{1}$ were obtained from the fit without any a-priori assumptions about the DW and the material structure.

\section{Linear approximation and the rule of thumb}

It is important to investigate as wide range of the field gradient $G$ values as possible, which is typically about one order of magnitude ${ }^{5,7}$ in our experiments. A very considerable decrease of the DW coercive field $H_{\mathrm{CW}}$ was observed within such a range, yet to determine the exact shape of the $H_{\mathrm{CW}}(G)$ functional dependence is difficult. The DW coercive field $H_{\mathrm{CW}}(G)$ dependence was approximated in Refs. 5 and 7 by a linear function as $H_{\mathrm{CW}}(G)=H_{\mathrm{CW}}(0)-a G$, where $H_{\mathrm{CW}}(0)$ was the coercive field of a free wall (not subjected to any gradient in the sample) and $a$ was a phenomenological proportionality constant which was related to the distribution of the coercivity-causing defects and to the actual domain geometry. However, the result of the DW coercive field $H_{\mathrm{CW}}(G)$ computation carried out in our much wider range of the field gradient values $G$ as shown in Fig. 5, is highly nonlinear. It is hyperbolic (22) in the limiting case of the pure WL process $(\alpha=0)$ or, more realistically it is given by the shapes obtained from the numerical simulations for finite $\alpha$ as described in Sec. II F in the present paper. However, in the narrow experimental range of the $G$ values, $x(G)$ can really be roughly approximated by a linear dependence similarly to results of Ref. 5, see Fig. 5. It also qualitatively corresponds to the expectations of the simple analysis published earlier in Ref. 4.

An experimental rule of thumb which connects the expectations on the DW coercive field $H_{\mathrm{CW}}$ values with the effective local field gradient, $G$, and with the sample domain structure quality (density), and which was pointed out in Ref. 5 follows also from the present results: The smaller the domain structure period (the larger the effective field gradient), the lower is the domain-wall coercive field (and the quasistatic hysteresis losses). This can be mathematically shown for actual regular periodic domain structures but the message is quite general and valid in any domain structure where the notion of its period has a reasonable meaning. This may give an important hint for tailoring soft magnetic devices towards decrease of coercive hysteresis losses. However, the existing inverse proportionality between the field gradient $G$ and the sample susceptibility implies also, that for achieving a de- crease of the DW coercive field $H_{\mathrm{CW}}$ by increasing of the field gradient $G$, the material in question must be operated at a decreased effective susceptibility and permeability. Evidently, an optimum has to be chosen depending on the actual technical task.

\section{CONCLUSIONS}

(1) The model proposed here gives a natural interpretation of the coercivity effects associated with the DW motion subjected to effective local field gradients, $G$, in a randomly nonunifom material. It predicts the DW coercive field, $H_{\mathrm{CW}}$, to be a decreasing function of the field gradient $G$, which is in agreement with both experimental observation ${ }^{5,7}$ and with expectations from a simple model with a periodic DW potential. ${ }^{4}$

(2) We applied a model description of the DW pinning field, $H_{p}$, described by the Wiener-Lévy stochastic process for an analysis of the coercive pinning of DW's in soft magnetic materials. The basic feature of the model is the description of the random DW pinning field, $H_{p}\left(x_{\mathrm{DW}}\right)$, by a set of coupled Langevin equations (12) containing two correlation lengths $\xi_{1}$ and $\xi_{2}$ such that (i) $H_{p}\left(x_{\mathrm{DW}}\right)$ is stationary for the DW displacements $\Delta x_{\mathrm{DW}} \gg \xi_{2}$; (ii) the slope $d H_{p} / d x_{\mathrm{DW}}$ controlling the DW susceptibility tends to be well defined when $\Delta x_{\text {DW }} \ll \xi_{1}$.

(3) Three coercive fields, $H_{\mathrm{DW}}, H_{\mathrm{dyn}}$, and $H_{\mathrm{CW}}$, were introduced. By a combination of analytical calculation methods and numerical simulation algorithms we estimated the total dynamic DW coercive field, $H_{\mathrm{DW}}$, associated with the given DW velocity averaged over the stochastic fluctuations, $\left\langle v_{\mathrm{DW}}\right\rangle$, and the static coercive field, $H_{\mathrm{CW}}$, representing the part of $H_{\mathrm{DW}}$ that survives in the quasistatic limiting condition: $H_{\mathrm{CW}}=\lim _{\left\langle v_{\mathrm{DW}}\right\rangle \rightarrow 0}\left(H_{\mathrm{DW}}-H_{\mathrm{dyn}}\right)$. In all cases we considered the coercive fields caused by pure DW pinning and depinning only, i.e., we worked with such a DW geometry, where any DW generation and/or annihilation, and any rotation of the magnetization vector within the domains could be neglected.

(4) The case when the stochastic pinning field, $H_{p}\left(x_{\mathrm{DW}}\right)$, follows a pure $\mathrm{WL}$ process characterized by $\left\langle\left|d H_{p}\right|^{2}\right\rangle=2\left(A_{H}^{2} / \xi_{2}\right) d x_{\text {DW }}$ (i.e., for the correlation lengths $\xi_{1} \rightarrow 0$ and $\left.\xi_{2} \rightarrow \infty\right)$, can be solved analytically but it is not very realistic. We obtained the simple hyperbolic relation between the DW coercive field and the field gradient, $H_{\mathrm{CW}}$ $\propto G^{-1}$, which qualitatively reproduces the experimentally observed decrease of the DW coercive field $H_{\mathrm{CW}}$ with increasing the field gradient $G$, but which yields an unrealistic divergence of $H_{\mathrm{CW}}$ at $G \rightarrow 0$ and relatively large values of $H_{\mathrm{CW}}$ at large field gradients, $G$.

(5) The general case of finite values of the correlation lengths, $\xi_{1}$ and $\xi_{2}$, was investigated by numerical simulations. In contrast to the hyperbolic solution and in agreement with the experimental observations, the DW coercive field $H_{\mathrm{CW}}$ was finite and approximately given by $A_{H}$ as $G$ approached zero, and $H_{\mathrm{CW}}$ decayed rapidly to zero for large field gradients, $G \gg A_{H} / \sqrt{\xi_{1} \xi_{2}}$.

(6) The dependencies of the DW pinning field on the field 
gradient $H_{\mathrm{CW}}(G)$ calculated for different $\alpha=\xi_{1} / \xi_{2}$ are similar. Therefore fitting of the computed $H_{\mathrm{CW}}(G)$ curves to any value $\alpha<1$ cannot answer the question of the best choice of $\alpha$. However, qualitative considerations suggest to prefer $\alpha=1$ and to expect $\xi_{1}=\xi_{2}=\xi$ to be comparable with the DW width parameter, $\delta_{w}=\sqrt{A / K_{u}}$. The agreement between $\xi$ and $\delta_{w}$ was found satisfactory in the two investigated samples. This result thus implies that the pinning field responsible for the DW coercivity in the investigated materials has a very fine structure and its interaction with DW is limited by the finite DW width.

\section{ACKNOWLEDGMENTS}

The authors are indebted to K. L. Metlov for his careful reading of the manuscript and many valuable remarks. This work was supported by the PECO Joint Research Project No. CIPA-CT93-0239 and Grant No. GACR No. 202/95/0022.
${ }^{1}$ H. R. Hilzinger and H. Kronmüller, J. Magn. Magn. Mater. 2, 11 (1976).

${ }^{2}$ H. Kronmüller, J. Magn. Magn. Mater. 24, 159 (1981).

${ }^{3}$ R. Schäfer, W. K. Ho, J. Yamasaki, A. Hubert, and F. B. Humphrey, IEEE Trans. Magn. 27, 3678 (1991).

${ }^{4}$ I. Tomáš, J. Magn. Magn. Mater. 87, 5 (1990).

${ }^{5}$ I. Tomáš, G. Vértesy, and K. L. Metlov, J. Magn. Magn. Mater. 150, 75 (1995).

${ }^{6}$ L. Půst and G. Bertotti, IEEE Trans. Magn. 30, 834 (1994).

${ }^{7}$ A. N. Grigorenko, S. A. Mishin, and E. G. Rudashevskii, Pisma Zh. Tech. Fiz. 14, 1772 (1988) [Fiz. Tverd. Tela 30, 2948 (1988)].

${ }^{8}$ G. Vértesy, I. Tomáš, and L. Půst, J. Phys. D 20, 1088 (1987).
${ }^{9}$ G. Vértesy, L. Bódis, I. Tomáš, and L. Půst, J. Phys. D 22, 1742 (1989).

${ }^{10}$ R. Vergne, J. C. Cotillard, and J. L. Porteseil, Rev. Phys. Appl. 16, 449 (1981).

${ }^{11}$ W. Grosse-Nobis, J. Magn. Magn. Mater. 4, 247 (1977).

${ }^{12}$ B. Alessandro, C. Beatrice, G. Bertotti, and A. Montorsi, J. Appl. Phys. 68, 2901 (1990).

${ }^{13}$ R. M. Josephs, in Magnetism and Magnetic Materials, edited by C. D. Graham and J. J. Ryne, AIP Conf. Proc. No. 10 (AIP, New York, 1972), p. 286.

${ }^{14}$ J. A. Seitchik, G. K. Goldberg, and W. D. Doyle, J. Appl. Phys. 42, 1272 (1971).

${ }^{15}$ A. Papoulis, Probability, Random Variables and Stochastic Processes (McGraw-Hill, New York, 1965). 